\title{
PDEparams: Parameter fitting toolbox for partial differential equations in Python
}

\author{
César Parra-Rojas and Esteban A. Hernandez-Vargas* \\ Frankfurt Institute for Advanced Studies, 60438 Frankfurt am Main, Germany
}

\begin{abstract}
Motivation: Partial differential equations (PDEs) is a well-established and powerful tool to simulate multi-cellular biological systems. However, available free tools for validation against data are not established. The PDEparams module provides flexible functionality in Python for parameter estimation in PDE models.

Results: The PDEparams module provides a flexible interface and readily accommodates different parameter analysis tools in PDE models such as computation of likelihood profiles, and parametric bootstrapping, along with direct visualisation of the results. To our knowledge, it is the first open, freely available tool for parameter fitting of PDE models.

Availability and implementation: The PDEparams module is distributed under the MIT license. The source code, usage instructions and step-by-step examples are freely available on GitHub at github.com/systemsmedicine/PDE_params.

Contact: vargas@fias.uni-frankfurt.de

\section{Introduction}

PDE models appear in a wide variety of biological contexts (Anderson et al. (2000); Jaeger et al. (2004); Reis et al. (2016); Hross and Hasenauer (2016)) and, while most available computational tools focus on the numerical integration of PDE models to varying degrees of efficiency and complexity - see, e.g., Guyer et al. (2009) or Alnæs et al. (2015) — we have not come across general-use implementations incorporating functionality for parameter optimisation with respect to data, and the analysis of parameter identifiability and variability. Moreover, a wide range of models in biology consist of simple equations, in simple spatial domains, and the data available for validation tends to be sparse. To the best of our knowledge, there is no publicly available, open and free-to-use tools for kinetic parameter estimation of PDE models, but only codes for specific examples mainly for ordinary differential equations (ODEs) - e.g., Nguyen and Hernandez-Vargas (2018). Here we present PDEparams, a free Python module for parameter fitting in PDE models, and the analysis of parameter estimates in a straightforward, intuitive manner.
\end{abstract}

\section{Materials and methods}

The PDEparams module is not only meant to work when all variables in the system are observed, but also in the more realistic case when data are available for only one or a few of them; additionally, we accommodate the case when the observed quantity corresponds to a function of the variables, rather than their raw values.

\subsection{The PDEmodel object}

The main component of the module is the PDEmodel object. As the input, the user provides the data (as a pandas (McKinney et al. (2010) ) DataFrame), along with the PDE model (as a function of the state vector), the initial condition (as a function of the coordinate vector), and the lower and upper bounds for the unknown parameters - the estimation is carried out using the Differential Evolution (DE) algorithm (Storn and Price (1997)), which performs constrained optimisation. Other arguments include the parameter names (used for tables and plots, defaults to 'parameter 1', 'parameter 2',...); the number of variables in the system (defaults to 1 ); the number of spatial dimensions - for completeness, the module can also handle ODEs, for which this value should be set to zero (defaults to 1); the number of replicates, defined as the number of different measurements per space-time coordinate (defaults to 1); the observed 
variable if not all are observed (all assumed observed by default); and the function to apply to the raw variables (defaults to None, and raw outputs are used).

The constructed object contains a time array and spatial grid-to which the initialising functions have been applied - for integration of the model. These and all other vector operations are carried out using NumPy (Van Der Walt et al. (2011)).

\subsection{Best fit parameters}

After construction of the PDEmodel object, parameters that provide the best fit between model and datawithin their specified bounds - can be obtained by simply running the fit() method. If no argument is provided, the function to be minimised will be the mean squared error (MSE). Other options include: i) the root mean squared error; ii-iii) the mean (and root mean) squared logarithmic error; iv) the mean absolute error; and v) the median absolute error. The errors are computed using scikit-learn's (Pedregosa et al. (2011)) built-in functions; the integration of the model itself is handled with SciPy (Jones et al. $(01))$, as is the DE optimisation.

When $\mathrm{fit}(\mathrm{)}$ is run, the best parameters and the lowest error are added as attributes to the PDEmodel object. The former are also printed to the screen.

\subsection{Likelihood profiles}

Likelihood profiles (Raue et al. (2009) ) are computed for each parameter by fixing its values on a predefined grid and re-estimating all the rest. This is done running the likelihood_profiles() method. When no argument is given, grid of size 100 is used as the default; for different grid sizes, the argument npoints may be used. If the best fit parameters have already been obtained, the error to be used for the likelihood profiles will match the one originally used with the fit() method. If not, the default mean squared error will be used. During estimation, a progress bar (da Costa-Luis et al. $(2019))$ is displayed on the screen.

As a result, a DataFrame with the parameter values and their corresponding error for each of the parameters is added as an attribute to the PDEmodel object. These results can then be used internally or be exported as, e.g., a .csv file.

\subsection{Bootstrapping}

Parametric bootstrapping is carried out by randomly choosing one replicate per space-time coordinate and re-estimating all parameters in multiple rounds. This is done with the method bootstrap (); the number of rounds is controlled by the argument nruns which, if not given, is assumed to be 100-note that, if only one measurement per space-time coordinate exists in the data, bootstrapping amounts to simply running the fit() method multiple times, and therefore has no effect. If the best fit parameters have already been obtained, the error to be used for bootstrapping will match the one originally used with the fit() method. If not, the default mean squared error will be used. During estimation, a progress bar (da Costa-Luis et al. (2019) ) is displayed on the screen.

As a result of the procedure, two DataFrame objects are added as attributes to the PDEmodel object: i) a statistical summary of the parameter values - printed to the screen; and ii) the raw results. These can then be used internally or be exported as, e.g., a .csv file.

\section{$2.5 \quad$ Visualisations}

Within the module, the likelihood profiles and the bootstrapping results can be directly visualised, respectively, using the methods plot_profiles() and plot_bootstrap(). These use Matplotlib (Hunter (2007)) and Seaborn (Waskom et al. (2017)). If the best fit parameters have already been obtained, they will be highlighted in the plots, as shown in Fig. 1 

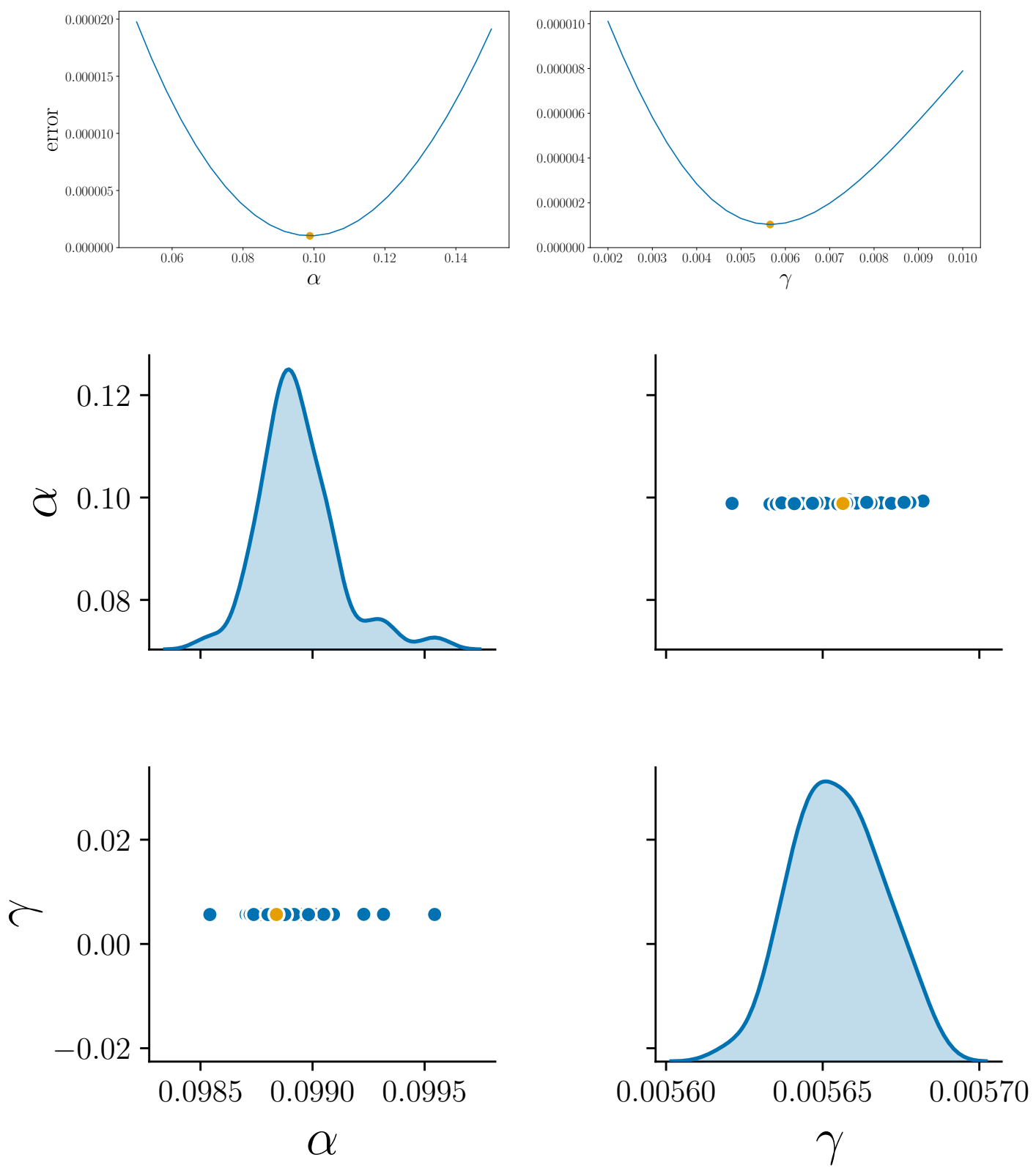

Figure 1: Likelihood profiles (top) and bootstrapping results (bottom) for the estimation of $\alpha$ and $\gamma$ from Eq. (1) using only the data for $m$. Visualisations obtained, respectively, with the plot_profiles() and plot_bootstrap() functions of PDEparams. Best fit parameters are shown in orange. Nominal values: $\alpha=0.1, \gamma=0.005$ 


\section{Example: tumour growth}

As an example use case, we take the two-dimensional, three-variable PDE model from Anderson et al. (2000), describing the dynamics of the invasion of host tissue by tumour cells:

$$
\begin{aligned}
\frac{\partial n}{\partial t} & =d_{n} \nabla^{2} n-\gamma \nabla \cdot(n \nabla f) \\
\frac{\partial f}{\partial t} & =-\eta m f \\
\frac{\partial m}{\partial t} & =d_{m} \nabla^{2} m+\alpha n-\beta m
\end{aligned}
$$

Here, $n$ corresponds to the tumour cells, $f$ to the host tissue, and $m$ to matrix-degradative enzymes associated with the tumour cells.

We start by integrating the model using the same parameters as in the paper $-d_{n}=0.001, d_{m}=0.001$, $\eta=10, \alpha=0.1, \gamma=0.005$, and $\beta=0$ - and the same initial condition for $n$ and $m$; the initial condition for the host tissue is heterogeneous and arbitrarily chosen - cf. Fig. 8 from the paper. After this, we generate artificial data by sampling the resulting dynamics on a $25 \times 25$ spatial grid at times $t=1,2, \ldots, 15$. We then use PDEparams to estimate the values of $\alpha$ and $\gamma$. The results are summarised in Fig. 1. for the case when only the data for $m$ are used for the estimation-i.e., $n$ and $f$ are assumed unobserved. The full step-by-step example is provided in the Supplementary Material, and is available in the module repository as a Jupyter notebook.

\section{Conclusions}

PDEparams is the first free module for the validation of PDE models against data and the analysis of their parameter estimates.

\section{Funding}

This work was supported by the Alfons und Gertrud Kassel-Stiftung and by the Deutsche Forschungsgemeinschaft (HE7707/5-1).

\section{References}

Alnæs, M. S., Blechta, J., Hake, J., Johansson, A., Kehlet, B., Logg, A., Richardson, C., Ring, J., Rognes, M. E., and Wells, G. N. (2015). The fenics project version 1.5. Archive of Numerical Software, 3(100).

Anderson, A. R., Chaplain, M. A., Newman, E. L., Steele, R. J., and Thompson, A. M. (2000). Mathematical modelling of tumour invasion and metastasis. Computational and mathematical methods in medicine, 2(2), $129-154$.

da Costa-Luis, C., L., S., Mary, H., Altendorf, K., noamraph, Korobov, M., Ivanov, I., Bargull, M., CHEN, G., mjstevens777, Pagel, M. D., James, Newey, C., Todd, Malmgren, S., Socialery, Nordlund, M., Zugnoni, M., McCracken, J., Hugo, Dill, F., Panteleit, D., Alexander, Rothberg, A., Fu, D., Bau, D., Persaud, A., Portnoy, A., Kottke, A., and Umer, A. (2019). tqdm/tqdm: tqdm v4.31.1 stable.

Guyer, J. E., Wheeler, D., and Warren, J. A. (2009). Fipy: Partial differential equations with python. Computing in Science \&s Engineering, 11(3), 6-15.

Hross, S. and Hasenauer, J. (2016). Analysis of cfse time-series data using division-, age-and label-structured population models. Bioinformatics, 32(15), 2321-2329.

Hunter, J. D. (2007). Matplotlib: A 2D graphics environment. Computing in Science Engineering, 9(3), 90-95.

Jaeger, J., Surkova, S., Blagov, M., Janssens, H., Kosman, D., Kozlov, K. N., Myasnikova, E., Vanario-Alonso, C. E., Samsonova, M., Sharp, D. H., et al. (2004). Dynamic control of positional information in the early drosophila embryo. Nature, 430(6997), 368. 
Jones, E., Oliphant, T., Peterson, P., et al. (2001-). SciPy: Open source scientific tools for Python.

McKinney, W. et al. (2010). Data structures for statistical computing in Python. In Proceedings of the 9th Python in Science Conference, volume 445, pages 51-56. Austin, TX.

Nguyen, V. K. and Hernandez-Vargas, E. A. (2018). Parameter estimation in mathematical models of viral infections using R. In Influenza Virus, pages 531-549. Springer.

Pedregosa, F., Varoquaux, G., Gramfort, A., Michel, V., Thirion, B., Grisel, O., Blondel, M., Prettenhofer, P., Weiss, R., Dubourg, V., et al. (2011). Scikit-learn: Machine learning in Python. Journal of machine learning research, 12(Oct), 2825-2830.

Raue, A., Kreutz, C., Maiwald, T., Bachmann, J., Schilling, M., Klingmüller, U., and Timmer, J. (2009). Structural and practical identifiability analysis of partially observed dynamical models by exploiting the profile likelihood. Bioinformatics, 25(15), 1923-1929.

Reis, R. F., dos Santos, R. W., and Lobosco, M. (2016). A plasma flow model in the interstitial tissue due to bacterial infection. In International Conference on Bioinformatics and Biomedical Engineering, pages 335-345. Springer.

Storn, R. and Price, K. (1997). Differential evolution-a simple and efficient heuristic for global optimization over continuous spaces. Journal of global optimization, 11(4), 341-359.

Van Der Walt, S., Colbert, S. C., and Varoquaux, G. (2011). The NumPy array: a structure for efficient numerical computation. Computing in Science ES Engineering, 13(2), 22.

Waskom, M., Botvinnik, O., O'Kane, D., Hobson, P., Lukauskas, S., Gemperline, D. C., Augspurger, T., Halchenko, Y., Cole, J. B., Warmenhoven, J., de Ruiter, J., Pye, C., Hoyer, S., Vanderplas, J., Villalba, S., Kunter, G., Quintero, E., Bachant, P., Martin, M., Meyer, K., Miles, A., Ram, Y., Yarkoni, T., Williams, M. L., Evans, C., Fitzgerald, C., Brian, Fonnesbeck, C., Lee, A., and Qalieh, A. (2017). mwaskom/seaborn: v0.8.1 (September 2017). 